\title{
Carboplatin/Paclitaxel/Pembrolizumab Regimen
}

National Cancer Institute

\section{Source}

National Cancer Institute. Carboplatin/Paclitaxel/Pembrolizumab Regimen. NCI

Thesaurus. Code C157396.

A regimen consisting of carboplatin, paclitaxel, and pembrolizumab that can be used in the treatment of metastatic squamous non-small cell lung cancer (NSCLC). 